\title{
Neural Network Modeling and Prediction of Surface Roughness in Machining Aluminum Alloys
}

\author{
N. Fang, P. Srinivasa Pai, N. Edwards \\ College of Engineering, Utah State University, Logan, USA \\ Email: ning.fang@usu.edu
}

Received 15 December 2015; accepted 10 May 2016; published 13 May 2016

\begin{abstract}
Artificial neural network is a powerful technique of computational intelligence and has been applied in a variety of fields such as engineering and computer science. This paper deals with the neural network modeling and prediction of surface roughness in machining aluminum alloys using data collected from both force and vibration sensors. Two neural network models, including a Multi-Layer Perceptron (MLP) model and a Radial Basis Function (RBF) model, were developed in the present study. Each model includes eight inputs and five outputs. The eight inputs include the cutting speed, the ratio of the feed rate to the tool-edge radius, cutting forces in three directions, and cutting vibrations in three directions. The five outputs are five surface roughness parameters. Described in detail is how training and test data were generated from real-world machining experiments that covered a wide range of cutting conditions. The results show that the MLP model provides significantly higher accuracy of prediction for surface roughness than does the RBF model.
\end{abstract}

\section{Keywords}

Artificial Neural Network, Modeling, Prediction, Surface Roughness, Machining, Aluminum Alloys

\section{Introduction}

As a powerful technique of computational intelligence, artificial neural network (ANN) has been applied in a variety of fields such as engineering and computer science [1]-[3]. This technique is particularity suitable for use in the modeling and prediction of complex engineering and computer science problems (or systems) where an analytical solution is not easily obtained due to the lack of relevant theories or the limitations of current understanding of the problems and systems. Among many other advantages, ANN can establish mathematical relationships among multiple input variables and multiple output variables.

In metal machining, an important operation in modern engineering manufacture, ANN has been employed to develop various models for tool condition monitoring and the prediction of machining performance measures, such as cutting forces, cutting vibrations, tool wear, tool life, and machined surface roughness [4]-[6]. For example, Dimla et al. [4] described various ANN techniques used for tool condition monitoring, especially for tool 
wear detection and identification. They concluded that multiple sensor inputs were important in order to improve the prediction accuracy of ANN models.

The present study deals with the neural network modeling and prediction of surface roughness in machining aluminum alloys using data collected from both force and vibration sensors. As a critical machining performance measure, surface roughness represents the machined surface quality and significantly affects the fatigue life of machined products [6] [7]. The overall goal of the present study is to determine which particular ANN technique-Multi-layer Perceptron (MLP) or Ra-dial Basis Function (RBF) - offers higher accuracy in predicting surface roughness in machining aluminum alloys. Both MLP and RBF are common neural net-work techniques with wide application in a variety of engineering and computer science fields.

The primary scientific contribution of the present study is that it takes into account the effect of tool-edge radius in machining for neural network modeling and prediction. Fang [8] has demonstrated that tool-edge radius has a profound effect in machining, especially in finish machining where the feed rate is at the same order of magnitude as the tool-edge radius. A substantial amount of experimental data by many other researchers [9]-[11] also demonstrates that tool-edge radius significantly affects cutting forces, cutting vibrations, machined surface roughness, and other machining performance measures. The critical effect of tool-edge radius has been neglected in the vast majority of existing studies that aimed to apply ANN for modeling and prediction in machining [4]-[6] [12]-[15]. The present study fills this important research gap.

The remainder of this paper is arranged in the following order. First, the inputs and outputs of the MLP and RBF models are described, followed by an introduction to how the two models were established. Then, described is how training and test data (for use in neural network modeling and prediction) were generated from machining experiments involving a wide range of cutting conditions. Experimental set-up and measurements are also described. Next, the results of MLP and RBF neural network modeling and prediction are presented and analyzed. Finally, conclusions are made at the end of the paper.

\section{MLP and RBF Neural Network Modeling}

\subsection{Inputs and Outputs of the Models}

In the present study, the MLP and RBF neural network models include the following eight inputs: 1) cutting speed $\mathrm{Vc}_{\mathrm{c}}(\mathrm{m} / \mathrm{min})$, 2) ratio of the feed rate to the tool-edge radius $\left.\mathrm{f} / \mathrm{rn}, 3\right)$ cutting force Fc $(\mathrm{N})$, 4) feed force Ff $(\mathrm{N}), 5)$ passive force $\mathrm{Fp}(\mathrm{N}), 6)$ cutting vibration in the direction of the cutting speed $\mathrm{Vx}(\mathrm{g}), 7)$ cutting vibration in the direction of the feed rate $\mathrm{Vy}(\mathrm{g})$, and 8) cutting vibration in the direction of the depth of cut $\mathrm{Vz}(\mathrm{g})$.

Note that for the second input listed above, the effect of the tool-edge radius in machining is represented by the ratio of the feed rate to the tool-edge radius. This is because the effect of the tool-edge radius depends on the feed rate employed in machining [6]. The tool-edge radius has a profound effect in machining if the feed rate is at the same order of magnitude as the tool-edge radius.

The outputs of the MLP and RBF models are five surface roughness parameters $(\mu \mathrm{m})$. The meaning of these surface roughness parameters has been well explained in relevant literature [16]. These five surface roughness parameters include: 1) average roughness Ra: arithmetic average of the absolute values of the roughness profile ordinates. Ra is the parameter most commonly used for evaluating surface roughness; 2) root-mean-square (RMS) roughness Rq: the root mean square average of the roughness profile ordinates; 3) peak roughness Rp: maximum peak height of the roughness profile ordinates; 4) maximum roughness height Rmax: maximum peak-to-valley height of the roughness profile ordinates; and 5) five-point average roughness Rz: five-point average peak-to-valley height of the roughness profile ordinates.

\subsection{Establishment of the MLP Model}

Based on a back-propagation algorithm, Multi-layer Perceptron (MLP) neural networks are among the most popular networks and have been widely applied in engineering research involving the modeling of metal machining [4] [6] [13] [14]. Mathematical details of the MLP network/model are available in the literature [17].

In the present study, computer codes for establishing the MLP model were developed using the MATLAB software package [18]. The number of hidden layers was set to be one. The number of neurons in the hidden layer was varied in order to develop a model that generalized well. The activation function used in the hidden neurons was logsig, a sigmoidal activation function. The output neuron had a linear activation function. Stan- 
dard built-in functions within MATLAB as well as trainrp and traingdm training functions were used for training the MLP model [18]. It was found that the trainrp algorithm was more efficient than traingdm, as the former took fewer epochs (within 20) and the latter required more than 10,000 epochs for reaching the set error level. Therefore, the trainrp algorithm was finally selected for training the MLP model in the present study.

\subsection{Establishment of the RBF Model}

Using a different mathematical method called curve fitting in a high dimensional space, Radial Basis Function (RBF) neural networks are a relatively new class of neural networks [19]. The primary advantage of the RBF model is its simplicity and the ease of implementation. The RBF network includes three quite different layers: input, hidden, and output layers. The transformation from the input layer to the hidden layer is non-linear; whereas the transformation from the hid-den layer to the output layer is linear [19].

In this study, computer codes for establishing the RBF model were also developed based on the MATLAB software package [18]. The function used for training the RBF model was newrb [18]. Based on trial and error, the spread (also called the width) of the RBF functions varied for different surface roughness parameters.

\section{Generation of Training and Test Data}

The MLP and RBF network models must be trained first using a set of training data. Then, the prediction accuracy of the two models can be validated using a set of test data. In the present study, all training and test data were generated from real-world machining experiments, rather than from virtual computer simulation experiments. A total of 45 sets of training and test data were generated, with each set including 13 data points, i.e., the eight in-puts and five outputs described in Section 2.1. Among these 45 sets, 38 sets (84\%) were randomly selected as training data, and the remaining 7 sets $(16 \%)$ were used as test data. The following two sub-sections describe the experimental set-up and measurements that were involved in data generation.

\subsection{Experimental Set-Up}

3D cutting experiments were conducted on a CNC turning center (HAAS SL10). The work material was a 2024-T351 aluminum alloy bar (ASTM B211 grade). This material has been widely applied in a variety of industries [7]. It has the following chemical compositions in percentage of weight: Al: 90.7 - 94.7; Cr: 0.1 max; Cu: 3.8 - 4.9; Fe: 0.5 max; Mg: 1.2 - 1.8; Mn: 0.3 - 0.9; Si: 0.5 max; Ti: 0.15 max; and Zn: 0.25 max.

Three coated carbide inserts, TPG432 KC8050 made by Kennametal Inc., were used. These tool inserts had different average tool-edge radii: $45.5 \mu \mathrm{m}, 54.7 \mu \mathrm{m}$, and $72.4 \mu \mathrm{m}$, respectively. They were carefully chosen to represent three different levels of tool-edge radius values in order to study their effects on surface roughness.

The cutting speeds varied at three levels: $150 \mathrm{~m} / \mathrm{min}, 250 \mathrm{~m} / \mathrm{min}$, and $350 \mathrm{~m} / \mathrm{min}$. The feed rates varied at five levels based on the ratio of the feed rate to the tool-edge radius: 1.0, 1.5, 2.0, 2.5, and 3.0. The depth of cut was kept constant at $0.8 \mathrm{~mm}$, the same as the tool nose radius. No coolants were used in the machining experiments in order to facilitate the experimental measurements of cutting forces, cutting vibrations, and machined surface roughness.

\subsection{Experimental Measurements}

Cutting forces were measured online for each experiment using a three-component quartz dynamometer (Kistler 9257B), a multi-channel dual-mode charge amplifier (Kistler 5010 B), and a computer data acquisition system (Labview). MATLAB was employed to filter the high-frequency noise from the collected signals. A MATLAB code was written to determine the average values of the three components of cutting forces, i.e., the cutting force $\mathrm{Fc}$, the feed force Ff, and the passive force Fp.

Cutting vibrations were simultaneously measured online for each machining experiment using a Triaxial ICP accelerometer (356A61) that was fixed to the tool holder. The accelerometer sensed the vibration signals in the $\mathrm{x}-, \mathrm{y}-$, and $\mathrm{z}$-directions, i.e., the cutting speed, feed rate, and depth of cut directions, respectively. The sensed vibration signals were sent to a low noise signal conditioner (PCB 482A22), an ICP accelerometer conditioning module (NI SCXI-1530/1531), and a computer for signal processing and display. The root mean square (RMS), i.e., the average of the squared values of the vibration amplitude, was calculated.

The machined surface roughness was measured offline after each machining experiment using a fine contour 
measuring instrument (Mitutoyo type-SV602). This instrument measured five surface roughness parameters: Ra, Rq, Rp, Rmax, and Rz. Three equally spaced locations around the circumference of the workpiece were involved in the measurement, and the average values of measurements were used.

\section{Results and Analysis}

\subsection{Training of MLP and RBF Models}

Figures 1-5 show the results of MLP and RBF training. As seen from these figures, both MLP and RBF models were well trained and reflect the fluctuation of training data generated under a wide range of cutting conditions.

\subsection{Testing of MLP and RBF Models}

Figures 6-10 show the comparisons of prediction accuracy between MLP and RBF models for each surface roughness parameter. As can be seen clearly, the pre-dictions by the MLP model is more close to the test data (i.e., experimentally-measured surface roughness parameters). Although the RBF model generates networks faster than the conventional training algorithms like traingdm used in MLP, the number of hidden neurons added by $\mathrm{RBF}$ to the network is nearly equal to the number of input patterns. This makes RBF a large network. In comparison, MLP generates a much more compact network and is able to generalize well on the test data.

Tables 1-4 further show the comparison of two primary surface roughness parameters, Ra and Rmax, between the MLP and RBF models. The mean squared error shown in these tables is defined as 1/2 (measured value - predicted value $)^{2}$. The smaller the mean squared error, the higher the prediction accuracy.

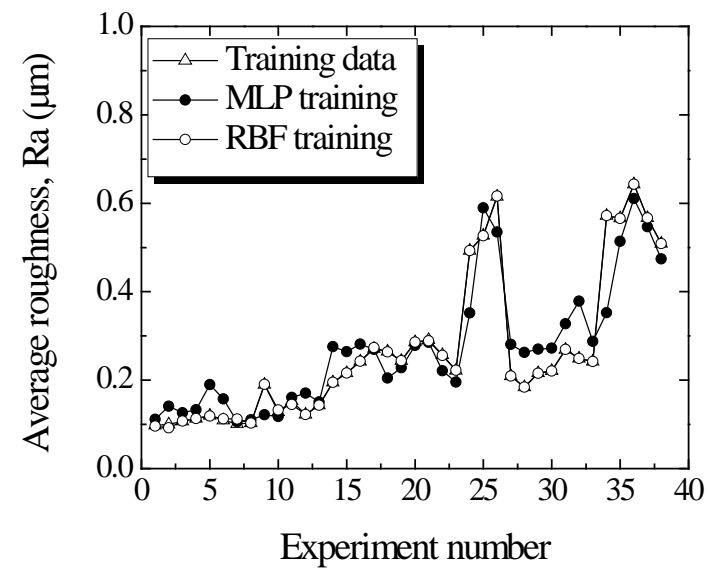

Figure 1. Training for average roughness Ra.

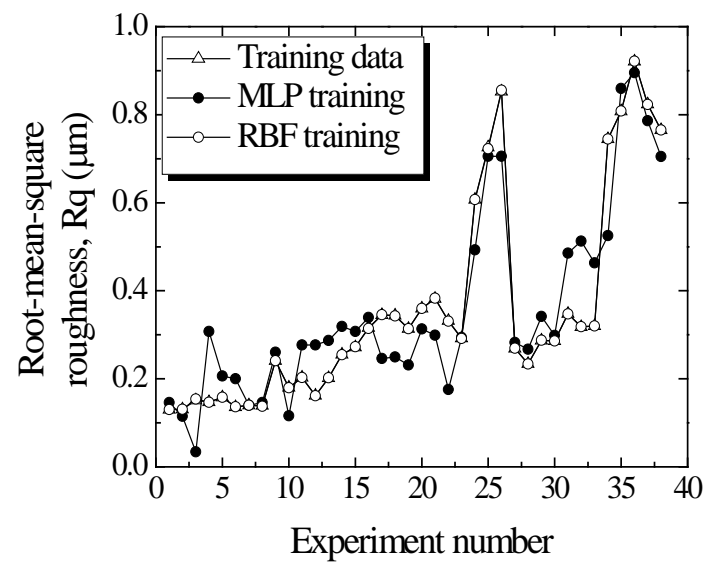

Figure 2. Training for root-mean-square roughness Rq. 


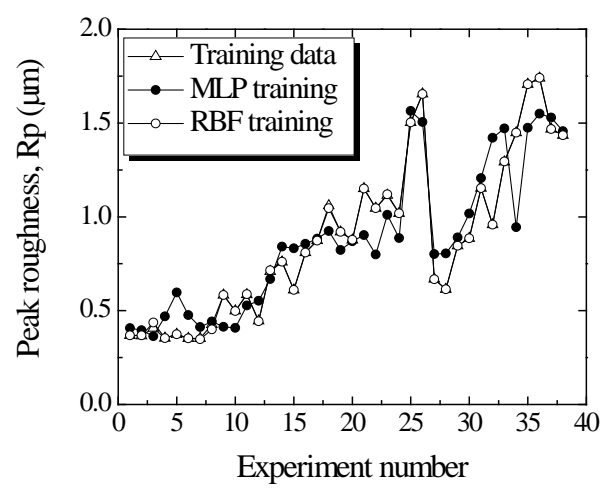

Figure 3. Training for for peak roughness Rp.

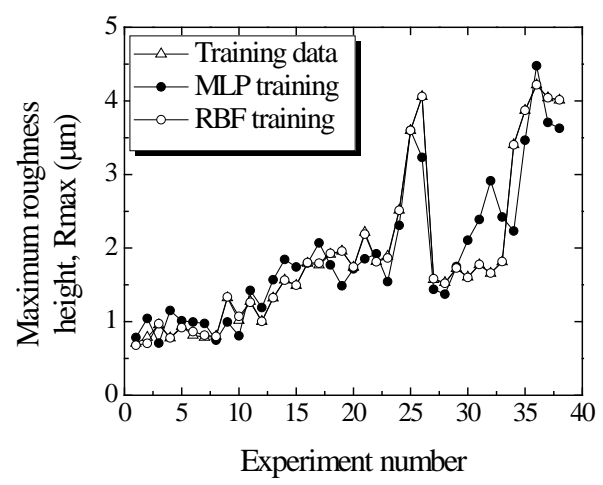

Figure 4. Training for maximum roughness height Rmax.

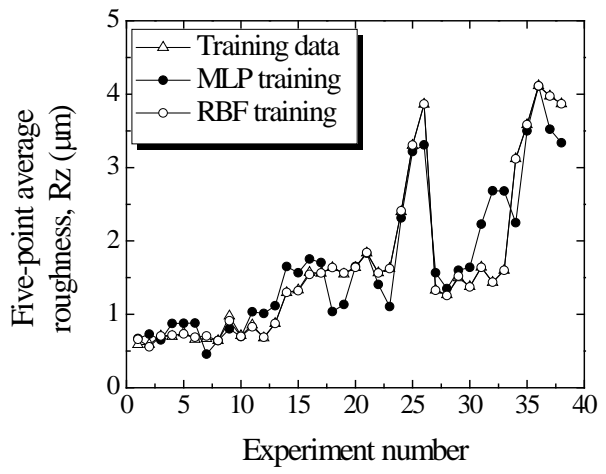

Figure 5. Training for five-point average roughness Rz.

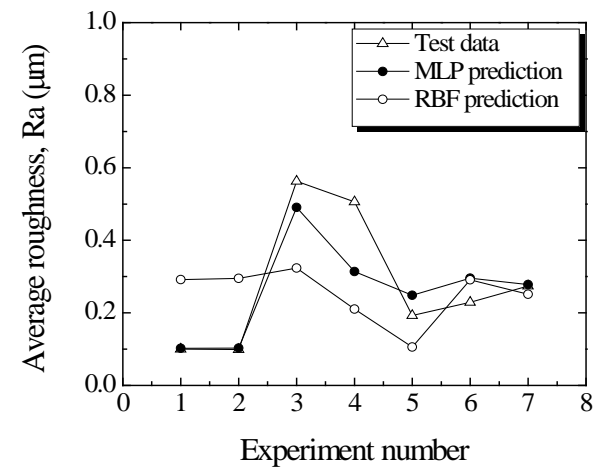

Figure 6. Prediction of average roughness Ra. 


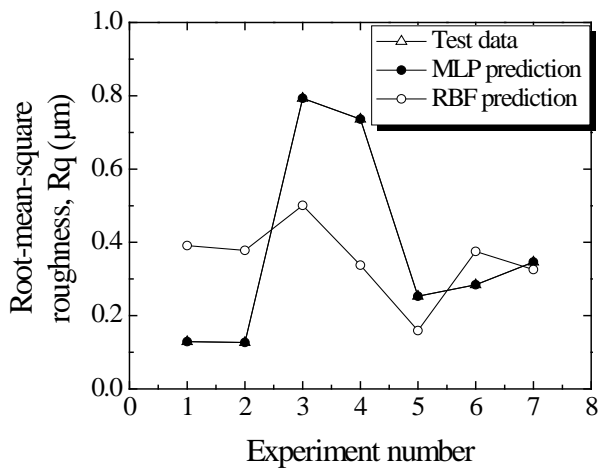

Figure 7. Prediction of root-mean-square roughness Rq.

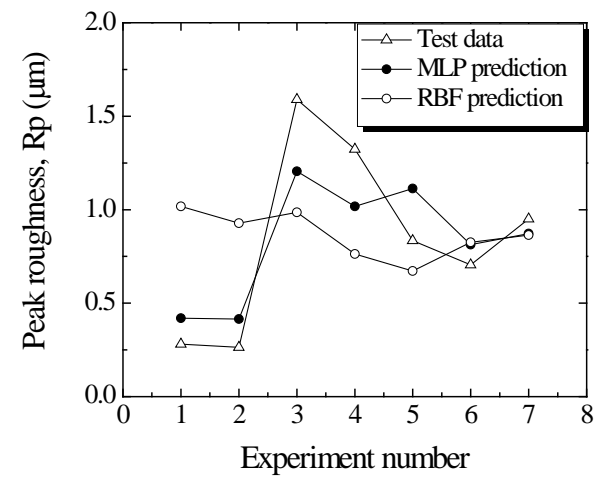

Figure 8. Prediction of peak roughness Rp.

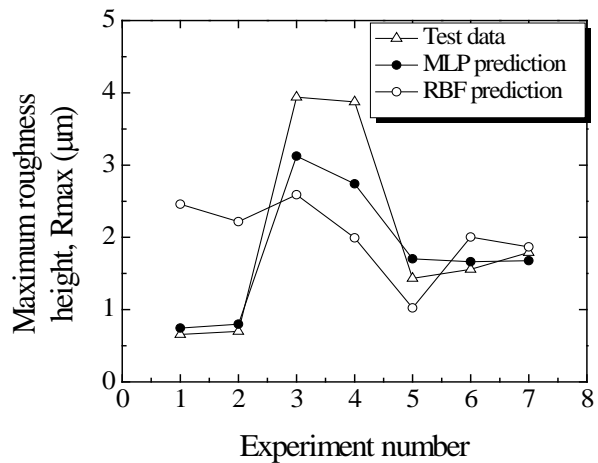

Figure 9. Prediction of maximum roughness height Rmax.

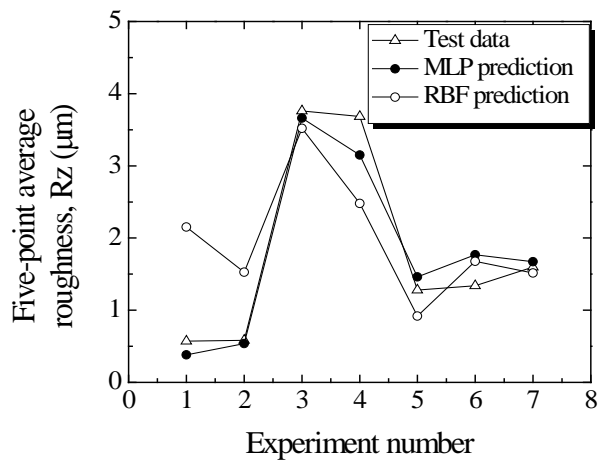

Figure 10. Prediction of five-point average roughness Rz. 
Table 1. Comparison of measured and predicted average roughness (Ra) for the MLP model.

\begin{tabular}{cccc}
\hline Experiment number & Measured & Predicted by MLP & Mean squared error \\
\hline 1 & 0.100 & 0.1022 & $2.42 \mathrm{E}-06$ \\
2 & 0.099 & 0.1028 & $7.22 \mathrm{E}-06$ \\
3 & 0.563 & 0.4909 & 0.002599 \\
4 & 0.506 & 0.3136 & 0.018509 \\
5 & 0.193 & 0.2485 & 0.001540 \\
6 & 0.229 & 0.2959 & 0.002238 \\
7 & 0.274 & 0.2778 & $7.22 \mathrm{E}-06$ \\
\hline
\end{tabular}

Table 2. Comparison of measured and predicted average roughness (Ra) for the RBF model.

\begin{tabular}{cccc}
\hline Experiment number & Measured & Predicted by RBF & Mean squared error \\
\hline 1 & 0.100 & 0.2919 & 0.018413 \\
2 & 0.099 & 0.2951 & 0.019228 \\
3 & 0.563 & 0.3232 & 0.028752 \\
4 & 0.506 & 0.2103 & 0.043719 \\
5 & 0.193 & 0.1056 & 0.003819 \\
6 & 0.229 & 0.2908 & 0.001910 \\
7 & 0.274 & 0.2511 & 0.000262 \\
\hline
\end{tabular}

Table 3. Comparison of measured and predicted maximum roughness height (Rmax) for the MLP model.

\begin{tabular}{cccc}
\hline Experiment number & Measured & Predicted by MLP & Mean squared error \\
\hline 1 & 0.657 & 0.7435 & 0.003741 \\
2 & 0.698 & 0.7995 & 0.005151 \\
3 & 3.940 & 3.1252 & 0.331950 \\
4 & 3.874 & 2.7394 & 0.643659 \\
5 & 1.433 & 1.7016 & 0.036073 \\
6 & 1.558 & 1.6623 & 0.005439 \\
7 & 1.791 & 1.6771 & 0.006487 \\
\hline
\end{tabular}

Table 4. Comparison of measured and predicted maximum roughness height (Rmax) for the RBF model.

\begin{tabular}{cccc}
\hline Experiment number & Measured & Predicted by RBF & Mean squared error \\
\hline 1 & 0.657 & 2.4589 & 1.623422 \\
2 & 0.698 & 2.216 & 1.152162 \\
3 & 3.940 & 2.5905 & 0.910575 \\
4 & 3.874 & 1.9925 & 1.770021 \\
5 & 1.433 & 1.0239 & 0.083681 \\
7 & 1.558 & 2.0052 & 0.099994 \\
\hline
\end{tabular}


Comparing the values of mean squared errors listed in Table $\mathbf{1}$ and Table $\mathbf{2}$ as well as those listed in Table $\mathbf{3}$ and Table 4, the MLP model has significantly higher prediction accuracy than does the RBF model, especially for the prediction of Rmax, i.e., maximum roughness height. The average mean squared errors (in\%) for both training and test data for five surface roughness parameters are: For the MLP model: Ra: 0.285\%; Rq: 0.205\%; Rp: 2.06\%; Rmax: 11.7\%; Rz: 6.12\%. For the RBF model: Ra: 0.83\%; Rq: 1.41\%; Rp: 6.11\%; Rmax: 40.3\%; Rz: $18.4 \%$.

\section{Conclusion}

This paper has described how a Multi-Layer Perceptron (MLP) and a Radial Basis Function (RBF) neural network model were developed to predict surface roughness in the machining of 2024-T351 aluminum alloy. The models take into account the effects of the tool-edge radius (via the ratio of the feed rate to the tool-edge radius), the cutting speed, cutting forces, and cutting vibrations on machined surface roughness. The results show that as compared to the RBF model, the MLP model offers significantly higher accuracy of prediction for machined surface roughness, especially for maximum roughness height. We suggested that the MLP model be used in the modeling and prediction of surface roughness in machining 2024-T351 aluminum alloy.

\section{References}

[1] Su, H. and Chong, K.T. (2007) Induction Machine Condition Monitoring Using Neural Network Modeling. IEEE Transactions on Industrial Electronics, 54, 2007, 241-249. http://dx.doi.org/10.1109/TIE.2006.888786

[2] Yousef, B.F., Knopf, G.K., Bordatchev, E.V. and Nikumb, S.K. (2003) Neural Network Modeling and Analysis of the Material Removal Process during Laser Machining. International Journal of Advanced Manufacturing Technology, 22, 41-53. http://dx.doi.org/10.1007/s00170-002-1441-9

[3] Pradhan, M.K., Das, R. and Biswas, C.K. (2009) Comparisons of Neural Network Models on Surface Roughness in Electrical Discharge Machining. Journal of Engineering Manufacture, 223, 801-808. http://dx.doi.org/10.1243/09544054JEM1367

[4] Dimla Jr., D.E., Lister, P.M. and Leighton, N.J. (1997) Neural Network Solutions to the Tool Condition Monitoring Problem in Metal Cutting-A Critical Review of Methods. International Journal of Machine Tools \& Manufacture, 37, 1219-1241. http://dx.doi.org/10.1016/S0890-6955(97)00020-5

[5] Lela, B., Bajic, D. and Jozic, S. (2009) Regression Analysis, Support Vector Machines, and Bayesian Neural Network Approaches to Modeling Surface Roughness in Face Milling. International Journal of Advanced Manufacturing Technology, 42, 1082-1088. http://dx.doi.org/10.1007/s00170-008-1678-z

[6] Ezugwu, E.O., Fadare, D.A., Bonney, J., Da Silva, R.B. and Sales, W.F. (2005) Modelling the Correlation between Cutting and Process Parameters in High-Speed Machining of Inconel 718 Alloy Using an Artificial Neural Network. International Journal of Machine Tools \& Manufacture, 45, 1375-1385. http://dx.doi.org/10.1016/j.ijmachtools.2005.02.004

[7] Jeelani, S. and Musial, M. (1986) Dependence of Fatigue Life on the Surface Integrity in the Machining of 2024-T351 Aluminum Alloy-Unlubricated Conditions. Journal of Materials Science, 21, 155-160. http://dx.doi.org/10.1007/BF01144714

[8] Fang, N. (2003) Slip-Line Modeling of Machining with a Rounded-Edge Tool, Part I: New Model and Theory. Journal of the Mechanics and Physics of Solids, 51, 715-742. http://dx.doi.org/10.1016/S0022-5096(02)00060-1

[9] Endres, W.J. and Kountanya, R.K. (2002) The Effects of Corner Radius and Edge Radius on Tool Flank Wear. Journal of Manufacturing Processes, 4, 89-96. http://dx.doi.org/10.1016/S1526-6125(02)70135-7

[10] Yang, K., Liang, Y.C., Zheng, K.N., Bai, Q.S. and Chen, W.Q. (2011) Tool Edge Radius Effect on Cutting Temperature in Micro-End-Milling Process. International Journal of Advanced Manufacturing Technology, 52, 905-912. http://dx.doi.org/10.1007/s00170-010-2795-z

[11] Denkena, B. and Biermann, D. (2014) Cutting Edge Geometries. CIRP Annals—Manufacturing Technology, 63, 631653. http://dx.doi.org/10.1016/j.cirp.2014.05.009

[12] Jeyakumar, S., Marimuthu, K. and Ramachandran, T. (2015) Optimization of Machining Parameters of AL6061 Composite to Minimize the Surface Roughness-Modelling Using RSM and ANN. Indian Journal of Engineering and Materials Sciences, 22, 29-37.

[13] Pal, S.K. and Chakraborty, D. (2005) Surface Roughness Prediction in Turning Using Artificial Neural Network. Neural Computing \& Applications, 14, 319-324. http://dx.doi.org/10.1007/s00521-005-0468-X

[14] Zhong, Z.W., Khoo, L.P. and Han, S.T. (2006) Prediction of Surface Roughness of Turned Surfaces Using Neural 
Networks. International Journal of Advanced Manufacturing Technology, 28, 688-693. http://dx.doi.org/10.1007/s00170-004-2429-4

[15] Feng, C.X.J., Yu, Z.G., Kingi, U. and Baig, M.P. (2005) Threefold vs. Fivefold Cross Validation in One-Hidden-Layer and Two-Hidden-Layer Predictive Neural Network Modeling of Machining Surface Roughness Data. Journal of Manufacturing Systems, 24, 93-107. http://dx.doi.org/10.1016/S0278-6125(05)80010-X

[16] DeGarmo, E.P., Black, J.T. and Kohser, R.A. (2003) Materials and Processes in Manufacturing. 9th Edition, John Wiley \& Sons Ltd., Chichester.

[17] Haykin, S. (1998) Neural Networks: A Comprehensive Foundation. 2nd Edition, Prentice Hall, Upper Saddle River.

[18] Beale, M.H., Hagan, M.T. and Demuth, H.B. (2015) Neural Network Toolbox User’s Guide. The MathWorks, Inc., Natick.

[19] Broomhead, D.S. and Lowe, D. (1988) Multi-Variable Functional Interpolation and Adaptive Networks. Complex Systems, 2, 327-355. 